\title{
Vollständigkeit und Qualität der Basisdaten und der Nachbeobachtung im Krebsregister
}

\author{
Eine Untersuchung am Beispiel des kolorektalen Karzinoms
}

S. Kropf ${ }^{1}$, E. Burger ${ }^{1}$, I. Radinski ${ }^{1}$, K. Ridwelski ${ }^{2,5}$, H. Lippert ${ }^{3,5}$, A. Altendorf-Hofmann ${ }^{4}$, J. Bernarding ${ }^{1}$

Institute

1 Institut für Biometrie und medizinische Informatik, Otto-von-Guericke-Universität, Magdeburg

2 Klinik für Allgemein- und Viszeralchirurgie, Klinikum Magdeburg gGmbH, Magdeburg

3 Klinik für Allgemein-, Viszeral- und Gefäßchirurgie, Otto-von-Guericke-Universität, Magdeburg

4 Klinik für Allgemein-, Viszeral- und Gefäßchirurgie, Universitätsklinikum Jena

5 An-Institut für Qualitätssicherung in der operativen Medizin gGmbH, Otto-von-Guericke-Universität Magdeburg

\section{Zusammenfassung}

Hintergrund und Fragestellung I In der Bundesrepublik Deutschland werden Tumorerkrankungen nicht nur in epidemiologischen, sondern auch in klinischen Registern erfasst, welche die Vernetzung der Behandlung, die Qualitätskontrolle und die klinische Forschung unterstützen. Voraussetzung dazu ist die in dieser Arbeit untersuchte Vollständigkeit der Erfassung.

Patienten und Methode I Wir verglichen die Daten aus dem klinischen Krebsregister Magdeburg und dem Register des An-Instituts für Qualitätssicherung in der operativen Medizin gGmbH an der Otto-von-Guericke-Universität Magdeburg. Anhand der erfassten Daten von Patienten mit kolorektalem Karzinom aus zwei großen Magdeburger Kliniken bewerteten wir den Erfassungsgrad bzw. die Vollständigkeit der Daten und such- ten nach systematischen Einflussfaktoren auf diese Kennziffern.

Ergebnisse I Von den im An-Institut erfassten Patienten waren 78,9\% im klinischen Krebsregister aufgeführt. Die Erfassungsrate verbesserte sich über die Zeit, hing aber auch von diagnostischen Kenngrößen wie dem Krebsstadium ab. Man erkennt Unterschiede zwischen beiden Registern, die auf ihre spezifischen Zielstellungen zurückgehen. Besonders in der Nachbeobachtung zeigten sich Gefahren für verfälschte Schätzungen aus den Registerdaten.

Folgerung | Die Sicherung von Vollzähligkeit, Vollständigkeit, Korrektheit und Aktualität der Erfassung der Daten ist eine wichtige Forderung an die Krebsregister, da Schätzungen von Qualitätsparametern wie Überlebenszeiten deutlich davon abhängen.

\section{Einleitung}

In der Bundesrepublik werden unter regional variierenden gesetzlichen Rahmenbedingungen Tumorerkrankungen in klinischen und epidemiologischen Krebsregistern erfasst. Während die epidemiologischen Register Daten für die Bestimmung wichtiger epidemiologischer Kennziffern in größeren Gebieten erfassen $[6,15]$, werden in den klinischen Krebsregistern Details der Diagnostik, der Therapie, der Behandlungsergebnisse sowie des Krankheitsverlaufes aufgeführt. Diese Daten sollen die vernetzte Behandlung der Tumorpatienten unterstützen und eine detaillierte Grundlage für Qualitätssicherung und klinische Forschung liefern [9, 7]. Das setzt eine hohe Vollzähligkeit, Vollständigkeit, Korrektheit und Aktualität der erhobenen Daten voraus.

Einen Hinweis auf die zu erwartenden Erkrankungszahlen liefert das Robert Koch-Institut unter Einbeziehung der Alters- und Geschlechtsstruktur des Einzugsgebietes [10]. Allerdings können so mögliche andere regionale Besonderheiten nicht vollständig berücksichtigt werden. Wir nutzten für unsere Studie die Tatsache, dass Patienten mit kolorektalem Karzinom aus zwei großen Magdeburger chirurgischen Kliniken über einen längeren Zeitraum in zwei unabhängigen
Registern mit unabhängigen Meldewegen erfasst wurden.

In mehreren Studien wurden bereits Analysen für Überlebenszeiten bei kolorektalem Karzinom anhand von Daten aus Krebsregistern durchgeführt $[11,12,8]$. Das hier betrachtete klinische Krebsregister Magdeburg (KKR) wurde 1993 an der Universität Magdeburg etabliert. Es erfasst Daten aus dem nördlichen Sachsen-Anhalt mit ca. 1,2 Millionen Einwohnern und leitet diese an das gemeinsame epidemiologische Register der neuen Bundesländer in Berlin weiter. Mit der Einführung der Meldepflicht in Sachsen-Anhalt im Jahr 2000 kann davon ausgegangen werden, dass eine stabile Meldetätigkeit erfolgt [5].

Das im Jahr 2000 gegründete An-Institut für Qualitätssicherung in der operativen Medizin gGmbH an der Otto-von-Guericke-Universität Magdeburg (An) verfolgt das Ziel, mittels prospektiver multizentrischer Beobachtungsstudien die Qualität der chirurgischen Behandlungen unter Routine-Bedingungen zu erfassen und zu analysieren, insbesondere auch für Patienten mit kolorektalem Karzinom [14, 13, 2].

Konkret sollten hier anhand der Patienten der zwei ausgewählten Kliniken die Daten des An- 
Instituts zur Kontrolle der Vollständigkeit der Erfassung der Tumorpatienten im klinischen Krebsregister genutzt werden.

Insbesondere werden folgende Fragen untersucht:

- Wie hoch ist die Rate der im An-Institut erfassten Patienten, die auch im klinischen Krebsregister gemeldet sind?

- Wie hoch ist die Übereinstimmung der an beide Register gemeldeten Patienten bzgl. der Vollständigkeit und Qualität der Daten?

- Gibt es zeitliche Trends oder andere Einflüsse auf die Vollständigkeit der Erfassung?

- Welchen Einfluss haben Erfassungsprobleme der Basisdaten und der Nachbeobachtung auf die Schätzungen der Überlebenszeiten und der Metastasierungsraten?

\section{Patienten und Methoden}

In unserer Untersuchung betrachteten wir Patienten mit kolorektalem Karzinom, die an der Klinik für Allgemein-, Viszeral- und Gefäßchirurgie der Otto-von-Guericke-Universität Magdeburg sowie an der Klinik für Allgemein- und Viszeralchirurgie am Klinikum Magdeburg gGmbH behandelt wurden. Beide Kliniken melden ihre Patienten an das KKR, sind aber zusätzlich Initiatoren und Teilnehmer der Qualitätssicherungsprogramme des An. Die Meldungen an das KKR bzw. das An umfassen einen ähnlichen Kern-Datensatz. Aber die beiden Register sind organisatorisch und in ihrer Zielstellung von einander unabhängig. Im An werden die Daten entweder über einen detaillierten Erhebungsbogen oder online durch Ärzte erfasst. Im KKR werden flächendeckend alle Tumorerkrankungen auf der Grundlage des Basisdatensatzes der Arbeitsgemeinschaft Deutscher Tumorzentren e.V. (ADT) mit speziellen organspezifischen Erweiterungen erfasst. Grundlage für die Erhebungen sind hier die Arztbriefe und ggf. Operationsberichte. Die Vollzähligkeit der Patienten mit kolorektalem Karzinom beträgt für das KKR derzeit ca. 70\% der zu erwartenden Fälle [10, 4]. Für diese Studie wurden die Daten des KKR in die Erfassungsstrukturen des An konvertiert, um eine Vergleichbarkeit herzustellen.

Es wurden alle Patienten einbezogen, die in den Jahren 2000 bis 2010 mit der Diagnose Kolonoder Rektumkarzinom in einer der beiden Kliniken behandelt und an das An gemeldet wurden. Patienten mit Kolonkarzinom in den Jahren 2005 bis 2007 wurden ausgeschlossen, da in diesen

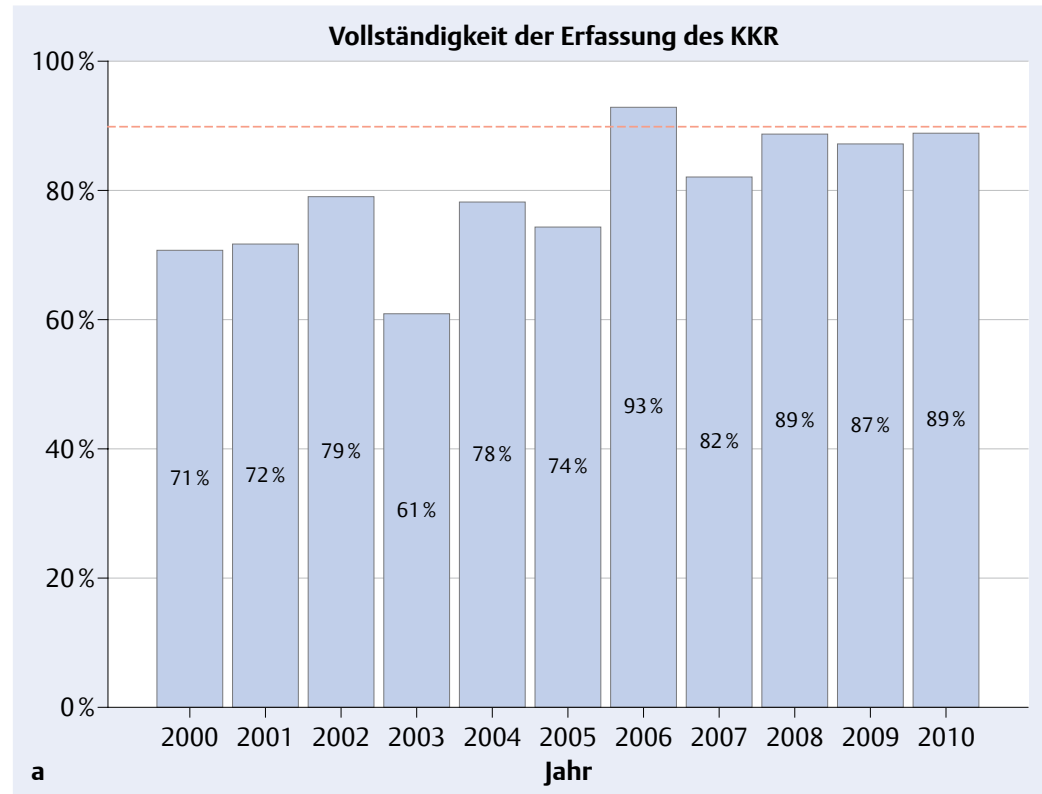

Vollständigkeit der Erfassung im KKR nach Klinik

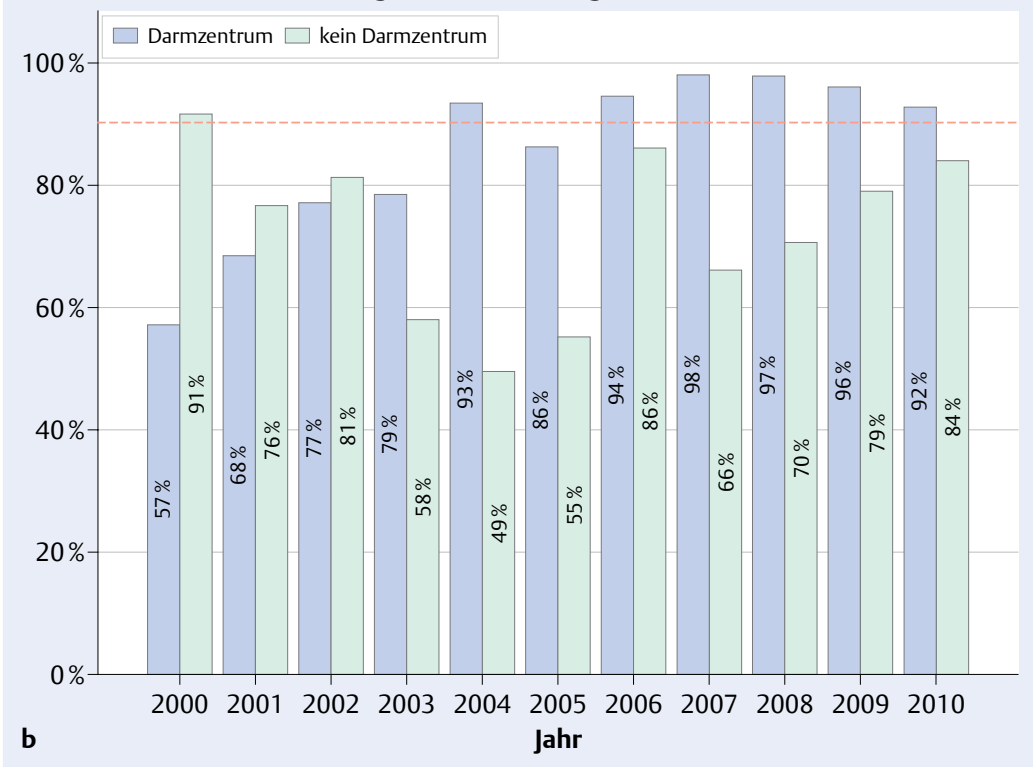

Abb. 1 Entwicklung der Melderaten des KKR über die Studienzeit. a) Beide

drei Jahren keine entsprechende Studie im An lief. Falls von einem Patienten mehrere Karzinome gemeldet waren, wurde bei zeitgleichen Karzinomen der jeweils schwerwiegendere Befund, ansonsten das zeitlich erste Karzinom ausgewählt. Ausgeschlossen wurden darüber hinaus 46 Patienten, deren Erstversorgung in einer auswärtigen Einrichtung erfolgte.

Das Zusammenführen der anonymisierten Daten aus beiden Registern erfolgte anhand des Geschlechts, der Diagnose sowie des Geburts- und Operationsdatums.

\begin{tabular}{|c|c|c|c|c|c|c|c|c|}
\hline & $\sigma^{x}$ & 우 & $<40$ ]. & $40-50 \mathrm{~J}$. & $50-60 \mathrm{~J}$. & $60-70 \mathrm{~J}$. & $70-80 \mathrm{~J}$. & $\geq 80 \mathrm{~J}$. \\
\hline gesamt & 1003 & 643 & 24 & 67 & 268 & 563 & 537 & 186 \\
\hline im KKR erfasst \% & 80,2 & 76,8 & 75,0 & 85,1 & 79,9 & 82,9 & 76,7 & 71,0 \\
\hline
\end{tabular}

Tab. 1 Melderaten des KKR in Abhängigkeit von Alter und Geschlecht der Patienten nach Angabe An. 

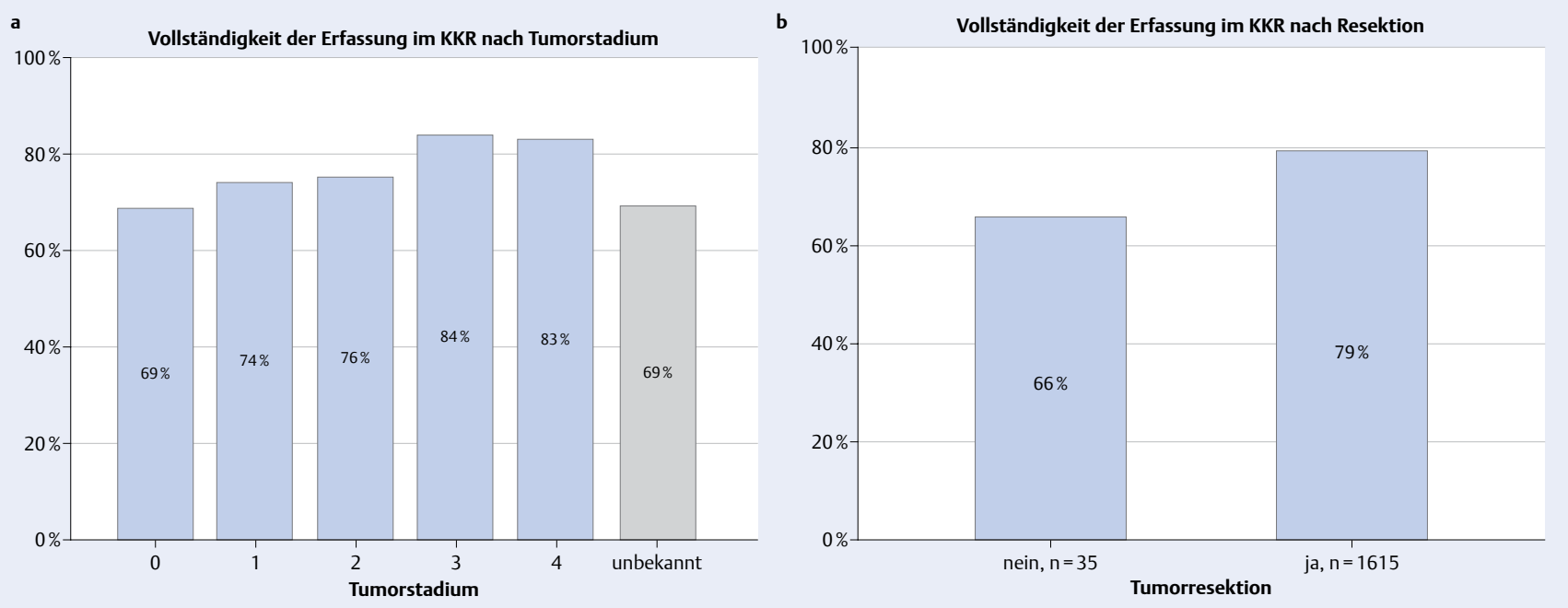

Abb. 2 Vollständigkeit des Registers in Abhängigkeit von a) Tumorstadium und $b$ ) Tumorresektion $(p=0,095)$. Die Klassifikation richtet sich dabei nach den Angaben des An.

Statistische Analysemethoden I Die Vollständigkeit der Erfassung im KKR wurde beurteilt, indem geprüft wurde, welcher Anteil der im An erfassten Patienten ebenfalls im KKR aufgeführt wurde. Vergleiche zwischen Subgruppen erfolgen mit dem $\mathrm{Chi}^{2}$-Test. Weiterhin wurden für die Patienten, die in beiden Registern erfasst waren, die aufgeführten Merkmalsausprägungen aus beiden Quellen ge-

Tab. 2 Gegenüberstellung der Einträge aus beiden Registern ( $n=1031$, X: nicht oder als unbekannt angegeben). Die prozentuale Übereinstimmung und Cohens Kappa (к) wurden unter Ausschluss der X-Stufen in beiden Registern berechnet.

\begin{tabular}{|c|c|c|c|c|c|}
\hline & & Ang & KKR & & \\
\hline pT-Kategorie & 1 & 2 & 3 & 4 & $x$ \\
\hline 0 & 0 & 0 & 0 & 0 & 1 \\
\hline 1 & 57 & 2 & 0 & 1 & 5 \\
\hline 2 & 2 & 166 & 5 & 0 & 6 \\
\hline 3 & 1 & 1 & 463 & 4 & 72 \\
\hline 4 & 0 & 2 & 4 & 187 & 33 \\
\hline$x$ & 4 & 1 & 2 & 1 & 11 \\
\hline & Über & רmuns & $\mathrm{K}, \mathrm{K}=0$ & & \\
\hline pN-Kategorie & 0 & 1 & 2 & & $x$ \\
\hline 0 & 470 & 3 & 2 & & 19 \\
\hline 1 & 2 & 221 & 3 & & 19 \\
\hline 2 & 7 & 6 & 232 & & 32 \\
\hline$x$ & 8 & 1 & 0 & & 6 \\
\hline & Über & רmuns & $\mathrm{K}, \mathrm{K}=0$ & & \\
\hline pM-Kategorie & 0 & 1 & & & \\
\hline 0 & 745 & 24 & & & \\
\hline 1 & 28 & 234 & & & \\
\hline & Über & רmunc & $\%, \mathrm{~K}=0$ & & \\
\hline R-Klassifikation & 0 & $>0$ & & & $x$ \\
\hline 0 & 725 & 56 & & & 15 \\
\hline$>0$ & 19 & 182 & & & 5 \\
\hline$x$ & 15 & 13 & & & 1 \\
\hline & Über & Imung & $\%, \mathrm{~K}=0$ & & \\
\hline
\end{tabular}

\section{Ergebnisse}

Allgemeine Angaben I Zwischen 2000 und 2010 wurden 1650 Patienten im An erfasst. Davon waren 1302 (78,9\%) auch im KKR registriert. Untersucht man den zeitlichen Verlauf der Erfassungsraten ( $\triangle$ Abb. $1 \mathrm{a}$ ), so stellt man eine deutliche Zunahme fest $(p<0,001)$. Allerdings unterschieden sich die beiden betrachteten Kliniken auch recht deutlich $(\checkmark$ Abb. 1 b).

In > Tab. 1 wird die Erfassungsrate in Abhängigkeit vom Alter und Geschlecht dargestellt. Die Erfassungsrate für Männer und Frauen unterschied sich nicht signifikant $(\mathrm{p}=0,106)$. Die Erfassungsraten hingen aber signifikant vom Alter des Patienten zum Zeitpunkt der Operation $a b(p=0,007)$. Sie waren am höchsten in der Gruppe von 40 bis 50 Jahren und am schlechtesten bei den Patienten ab 80 Jahren.

Diagnostik und Therapie | Exemplarisch wird in - Abb. 2 die Vollständigkeit der Erfassung in Abhängigkeit vom Tumorstadium und von der Durchführung der Resektion (beides nach den Angaben des An) betrachtet. Man erkennt eine statistisch signifikante Zunahme der Erfassungsrate mit steigendem Stadium $(p<0,001$, sowohl mit als auch ohne Einbezug des unbekannten Stadiums). Weiterhin deutete sich eine verminderte 
Erfassung der Patienten ohne erfolgte Resektion an $(\mathrm{p}=0,095)$.

Der folgende Vergleich berücksichtigt nur Patienten, die in beiden Registern erfasst sind. Weiterhin werden

- In-Situ-Karzinome (pTis),

- neuroendokrine Tumoren (NET),

- gastrointestinale Stromatumore (GIST),

- Melanome,

- Sarkome und

- Lymphome

ausgeschlossen. Nicht berücksichtigt wurden weiterhin Patienten, bei denen der Primärtumor nicht oder lediglich durch lokale Exzision entfernt wurde, sowie Patienten mit neoadjuvanter Therapie, da bei diesen die pTNM-Klassifikation nicht oder nur unvollständig erfolgen kann. Damit verblieben 1031 Patienten.

- Tab. 2 zeigt die Übereinstimmung der beiden Datenquellen für die pathologische TNM-Klassifikation und für das Resektionsergebnis. Für die $\mathrm{T}-, \mathrm{N}-$ und M-Kategorie stimmten die Angaben in mindestens $95 \%$ überein ( $\mathrm{k}$-Werte über 0,86). Die R0-Spezifikation zeigte 92,4\% Übereinstimmung mit $\mathrm{K}=0,78$. Allerdings fanden sich bei $\mathrm{T}$ - und $\mathrm{N}$-Kategorie in beiden Registern zusammen 13\% bzw. 8\% fehlende Angaben, beim R0-Status 5\%. Der größere Teil der Fehlwerte stammt dabei aus dem KKR als Hinweis auf mangelnde Qualität der Datenübermittlung durch Arztbriefe.

Fehlende Angaben bei N- und T-Kategorie führen zwangsläufig zu unterschiedlicher Stadieneinteilung, wobei sich die Effekte summieren. Bei 943 der 1031 Patienten (92\%) stimmte die Stadieneinteilung überein. Schließt man wieder die Patienten mit fehlender Angabe in einer der beiden Datenquellen aus, erhält man eine prozentuale Übereinstimmung von $94 \%$ bei einem $\mathrm{k}$ von 0,918 .

Nachbeobachtung I Wir betrachten eine Nachbeobachtung als adäquat, wenn der Patient entweder 5 Jahre oder bis zum Jahr 2011 beobachtet wurde oder verstorben ist. Betrachtet man die oben ausgewählten 938 Patienten mit übereinstimmendem Stadium I-IV unter diesem Aspekt, so zeigt sich, dass im KKR in 73,2\% der Fälle eine adäquate Nachbeobachtung erfasst wurde, im An nur in 30,7\% ( $\mathrm{p}<0,001)$. $>$ Abb. 3 zeigt die Entwicklung dieser Kenngröße über die Zeit. Während im KKR in den letzten Jahren die angestrebte Rate von mindestens $80 \%$ erreicht wurde, liegen die Angaben im An deutlich darunter. Die Verteilung der auf 60 Monate (5 Jahre) beschränkten Nachbeobachtungszeiten von KKR und An ist in - Abb. 4 dargestellt. Im KKR gab es einen großen Anteil von Patienten, welche die Nachbeobachtungszeit von 5 Jahren erreichten. Die mediane Nachbeobachtungszeit beträgt im KKR 25,1 Monate, im An 3,2 Monate ( $\mathrm{p}<0,001)$.

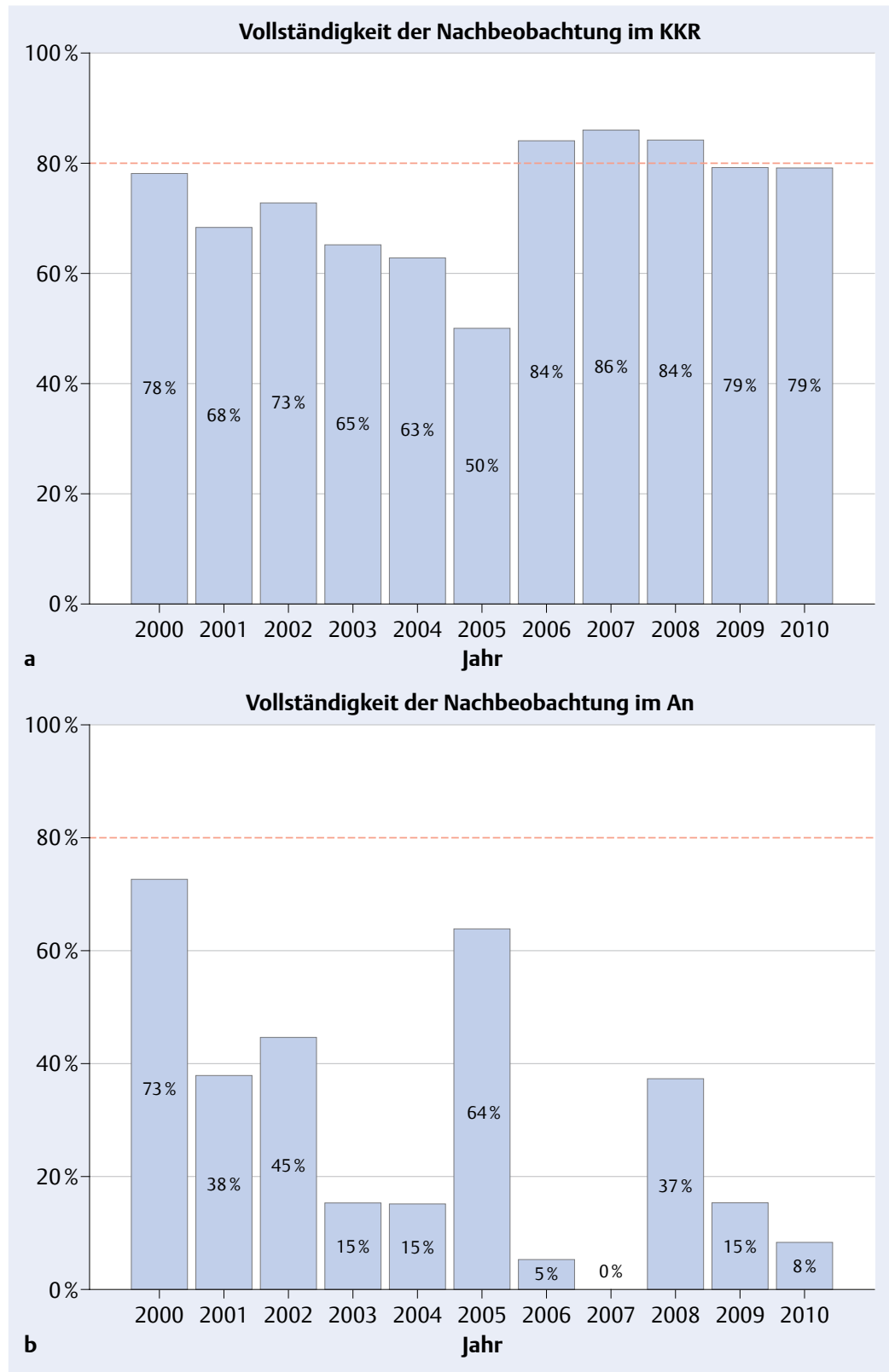

Abb. 3 Vollständigkeit der Nachbeobachtung a) im KKR sowie b) im An, jeweils $n=938$.

Dementsprechend unterschieden sich auch die Langzeitergebnisse. Nach den Angaben des An sind Rot: $80 \%$. in den ersten 5 Jahren $22,6 \%$ der Patienten verstor-

ben, nach den Angaben des KKR 38,7\% ( $p<0,001$ ).

Es stellt sich die Frage, inwieweit die bislang beobachteten Unterschiede der beiden Datenquellen die Schätzungen der Überlebenszeitkurven als wichtigen Indikator der Ergebnisqualität beeinflussen. Um das zu untersuchen, wurden zunächst die Kaplan-Meier-Schätzungen der Überlebenszeitkurven aus dem KKR und dem An separat bestimmt, jeweils anhand der aus der jeweiligen Quelle eingeschlossenen Patienten. $>$ Abb. 5 zeigt die beiden auf 5 Jahre begrenzten Kurven, einmal insgesamt, einmal stratifiziert nach den Stadien IIV bzw. X (wenige Fälle mit Stadium 0 wurden hier ausgelassen). Die Schätzungen aus KKR und An stimmten hier jeweils recht gut überein, nur bei unbekanntem Stadium gab es stärkere Abweichungen. 

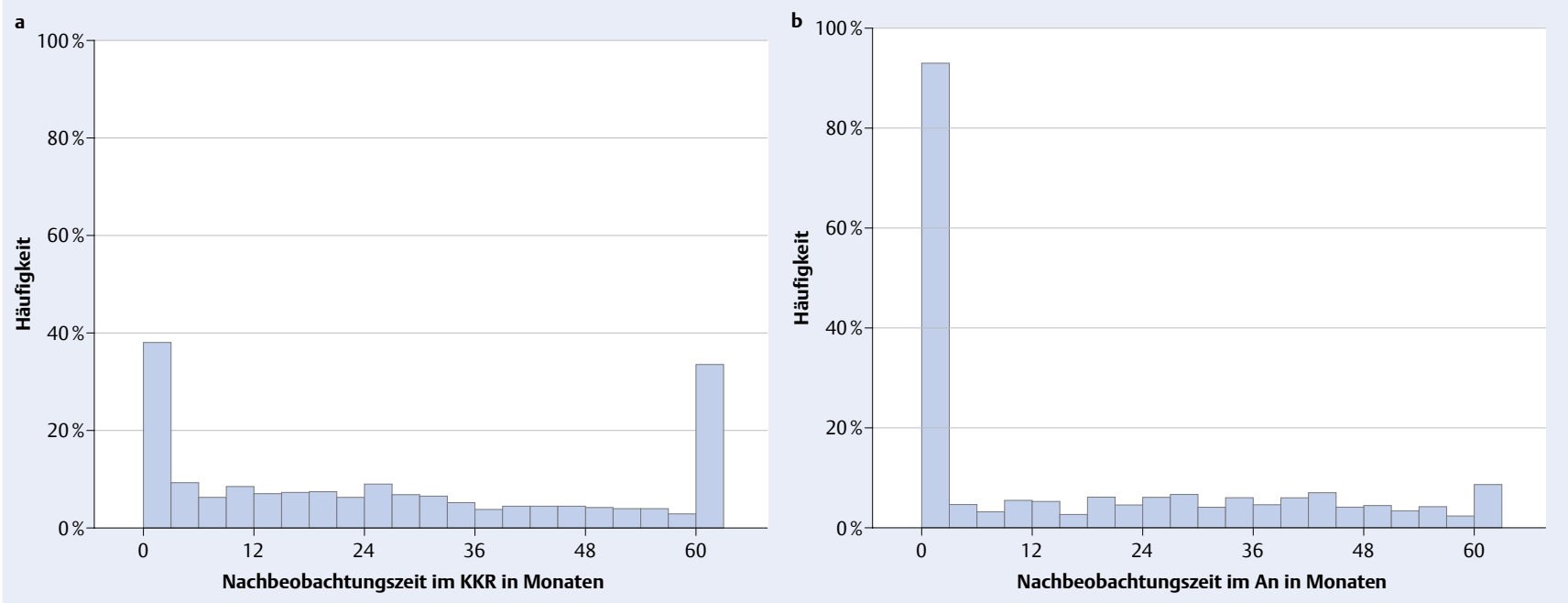

Abb. 4 Verteilung der Nachbeobachtungszeit a) im KKR sowie b) im An, jeweils $\mathrm{n}=938$.

Abb. 5 Separate Kaplan-MeierSchätzungen der Überlebenszeitkurven mit allen verfügbaren Patienten; a) unstratifiziert, b) stratifiziert nach Tumorstadium.
Das ändert sich, möglicherweise auch wegen des reduzierten Stichprobenumfanges, wenn man nur die Rektumkarzinome betrachtet ( $\bullet$ Abb. 6). Hier wurden fast alle Abweichungen zwischen KKR und An größer.

Diese Unterschiede verschwanden weitgehend, wenn die Kurven jeweils mit den gleichen Patienten mit bekannter und übereinstimmender Stadieneinteilung zwischen beiden Datenquellen berechnet wurden ( $\triangleright$ Abb. 7). Es blieben aber selbst in dieser Selektion Unterschiede zwischen beiden Kurven bestehen, was besonders in der unstratifizierten Darstellung im Bereich über zwei Jahre sichtbar wird.

Auf statistische Tests zum Vergleich der Überlebenszeitkurven wird hier verzichtet, da die zu vergleichenden Kurven teils aus abhängigen, teils aus unabhängigen Stichproben stammen.

\section{Diskussion}

Die Problematik unvollzähliger, unvollständiger, unkorrekter oder nicht aktueller Datenerfassung rückt zunehmend in das Bewusstsein der Registerbetreiber. Einerseits werden die Bemühungen um eine bessere Erfassung der Daten forciert, andererseits wird aber auch kritisch untersucht, welchen Einfluss die bestehenden Mängel auf die Analyse-Ergebnisse haben können.

Jüngere Untersuchungen betrachten z.B. durch simulierte Fehlwerte in vorhandenen Daten die Leistung verschiedener Imputationsverfahren [3]. Solche Verfahren benutzen üblicherweise die Missing-at-Random-Annahme. Diese besagt sinngemäß, dass mögliche systematische Einflüsse auf die Ausfallwahrscheinlichkeit durch andere vorhandene Angaben vom Patienten berücksichtigt und korrigiert werden können. Diese Annahme ist praktisch kaum zu überprüfen. Weiterhin muss man damit rechnen, dass bestimmte Teilgruppen der Patienten mit über- oder unterdurchschnittlicher Wahrscheinlichkeit gar nicht erfasst werden, so dass auch keine Ersetzung einzelner fehlender Angaben erfolgen kann. Wir nutzten daher in unserem Umfeld die besondere Situation, dass wir für ausgewählte Entitäten eine Registrierung der Tumorfälle in zwei unterschiedlichen Erfassungssystemen mit relativ unabhängigen Meldewegen haben.
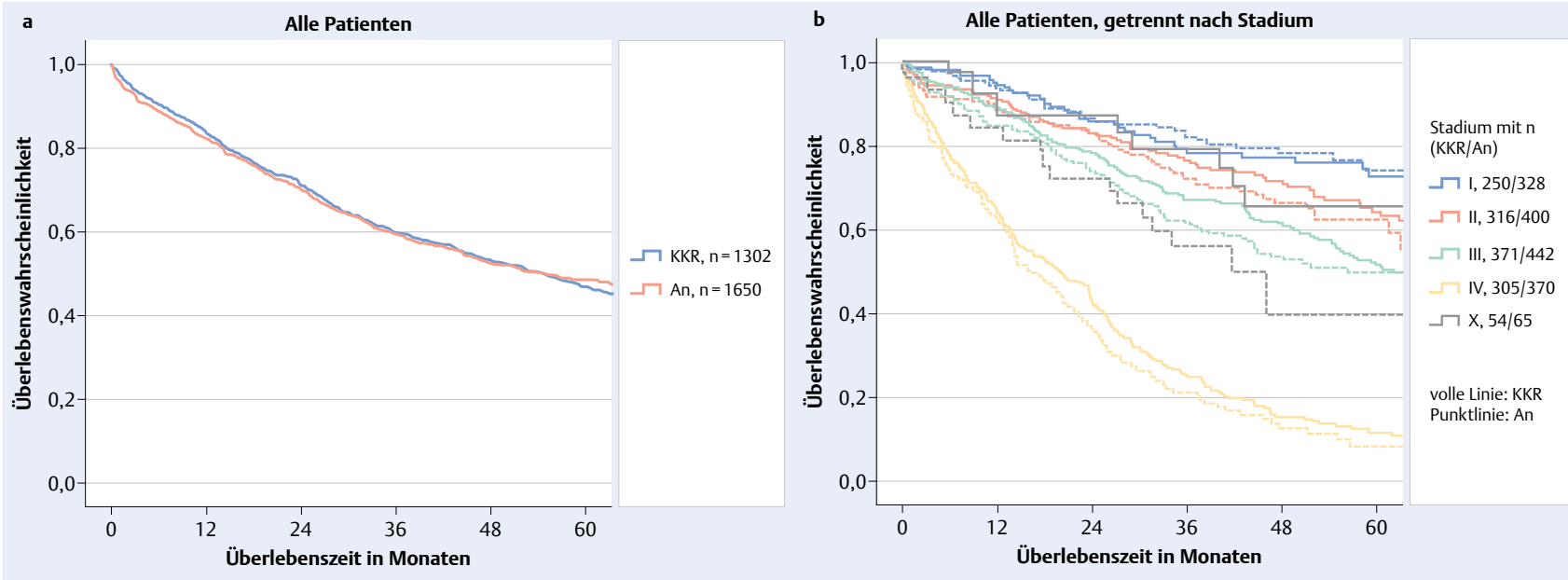
Im KKR fehlte etwa ein Sechstel der Patienten des An, wobei sich die Erfassungsrate über die Zeit verbesserte. Dabei bestand eine Abhängigkeit vom Alter, wobei die Altersgruppe ab 80 Jahren am schlechtesten erfasst wurde. Es zeigte sich hier weiterhin, dass die Zertifizierung einer Klinik als Darmzentrum ein wichtiger Stimulus für die Erzielung einer hohen Melderate war.

Naturgemäß können wir keine direkten Aussagen darüber treffen, wie viele Patienten zwar zur untersuchten Population der beiden Kliniken gehören, aber in keinem der beiden Register erfasst wurden. Wir vermuten aber, dass dieser Anteil klein ist. Das wird auch aus dem Vergleich mit epidemiologischen Daten unterstützt, wonach das KKR etwa eine Erfassung von $70 \%$ aufwies, während $79 \%$ aller An-Patienten im KKR wiedergefunden wurden.

Bei den diagnostischen Angaben und der Angabe des erzielten R-Status konnte eine gute, wenn auch nicht vollständige, Übereinstimmung der Einträge festgestellt werden. Es zeigte sich, dass mit zunehmender Krankheitsschwere die Patienten mit höherer Wahrscheinlichkeit im KKR erfasst wurden. Das lässt sich gut daraus erklären, dass dies auch mit einer zunehmenden Anzahl von Arztkontakten und damit einer höheren Wahrscheinlichkeit der Meldung an das KKR verbunden ist. Beim An erfolgen die Meldungen weitgehend über die beteiligten chirurgischen Einrichtungen selber. Aber auch wenn ein Patient in beiden Registern erfasst ist, gab es einen beträchtlichen Anteil von fehlenden Angaben in einigen der hier betrachteten diagnostischen Kategorien.

Eine kontinuierliche Nachbeobachtung erfordert einen großen Aufwand, ist jedoch zur Beurteilung der Ergebnisqualität unverzichtbar. Auf Probleme, die durch eine unvollständige Nachbeobachtung entstehen können, wurde bereits in [1] hingewiesen.

Das KKR ist bei der Erfassung der Langzeitverläufe durch die Nutzung der vernetzten Arztkontakte im Vorteil. Weiterhin erhält das KKR vierteljährlich über das epidemiologische Krebsregister einen Abgleich der Todesfälle, sodass diese im KKR weitgehend erfasst werden. Von den noch lebenden Patienten ist bei einem hohen Anteil die maximal mögliche Länge der Nachbeobachtung erreicht. Ein Teil der im KKR fehlenden Nachbeobachtung-Angaben für eine längere Nachbeobachtungsdauer erklärt sich aus der Tatsache, dass das KKR Magdeburg zwar ein regionales Register ist, aber auch etwa $10 \%$ Patienten mit einem Wohnsitz außerhalb des Erfassungsgebietes umfasst. Außerdem befand sich in der Gruppe mit kurzer Nachbeobachtung ca. ein Drittel der Patienten im Stadium 1.

Einer der Gründe für eine fehlende Nachbeobachtung im An ist die fehlende Zustimmung der Pa-

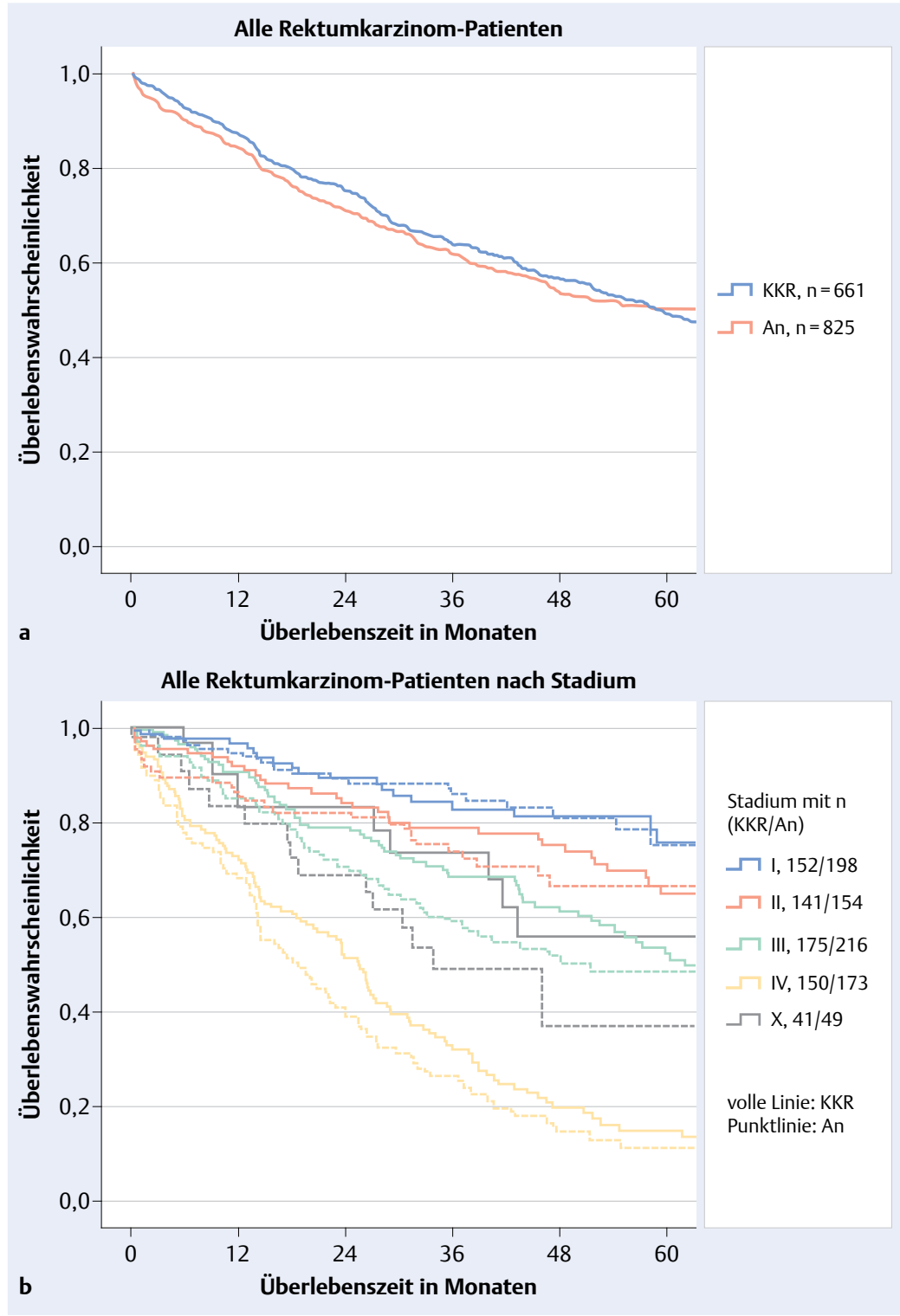

Abb. 6 Separate Kaplan-MeierSchätzungen der Überlebenszeitkurven mit jeweils allen Rektumkarzinom-Patienten der beiden Datenquellen; a) unstratifiziert, b) stratifiziert nach dem Tumorstadium. 


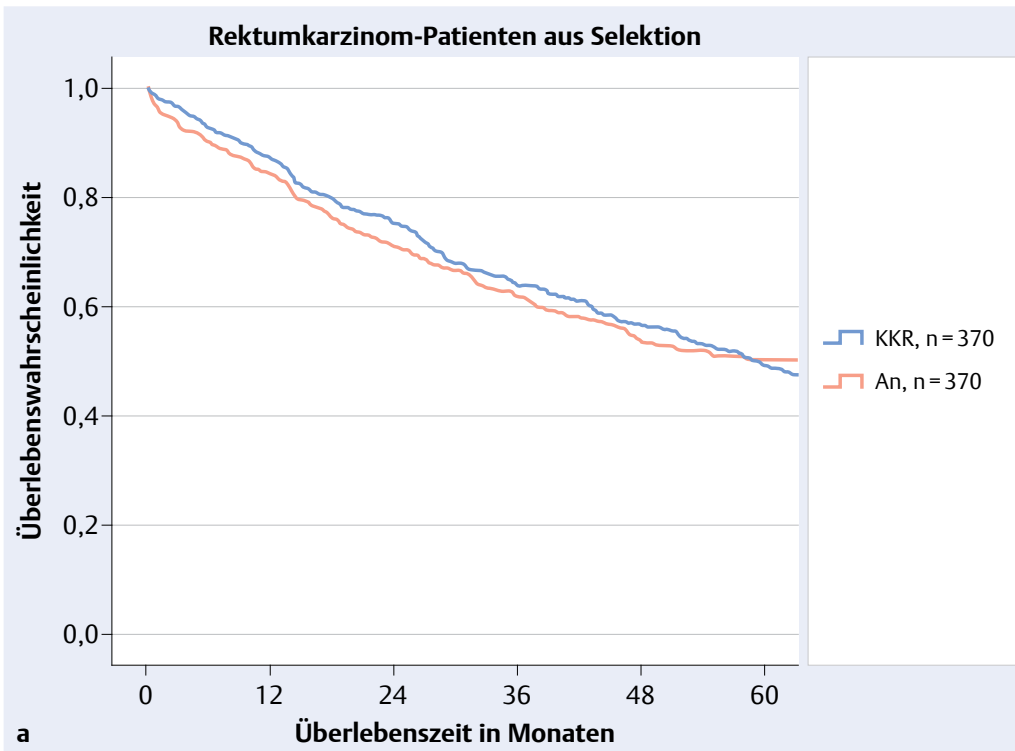

Rektumkarzinom-Patienten aus Selektion nach Stadium

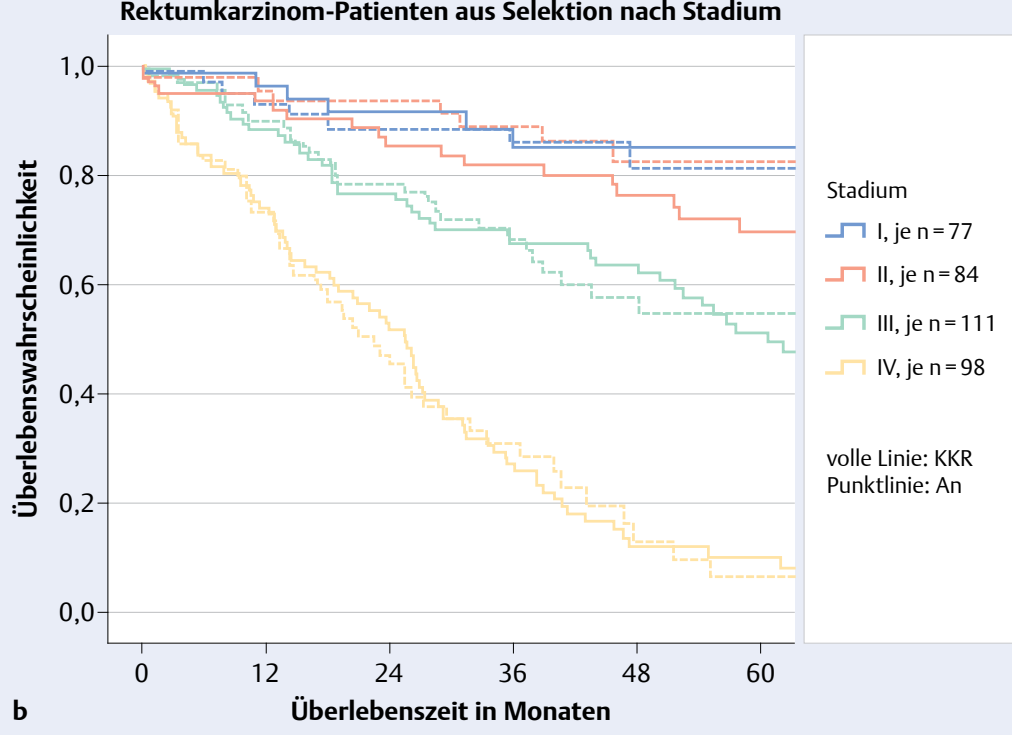

Abb. 7 Separate Kaplan-Meier-Schätzungen der Überlebenszeitkurven mit den Rektumkarzinom-Patienten der Auswahlmenge aus beiden Datenquellen; a) unstratifiziert, b) stratifiziert nach dem Tumorstadium.

\section{Folgerung}

Die Qualitätsanalyse der Behandlung von Krebskranken ist das erklärte Ziel der klinischen Krebsregister. Hierzu ist es wichtig, alle Aufmerksamkeit auf die Vollzähligkeit, Vollständigkeit, Richtigkeit und Aktualität der Daten zu richten, aber auch die Limitierungen zu kennen. Dies ist eng mit dem Engagement der Diagnostiker und Behandler für das zuständige klinische Krebsregister verknüpft. Trotz aller Bemühungen haben klinische Krebsregister nach wie vor Schwierigkeiten, die relevanten Daten von Tumorpatienten vollzählig, vollständig und korrekt zu erfassen.

Insbesondere ist die Sicherung der LangzeitNachbeobachtung mit großen logistischen Herausforderungen verbunden. Die systematische Erhebung von Längsschnittsdaten und der Datenaustausch mit anderen Registern werden an vielen Stellen zudem von rigiden Datenschutzbestimmungen limitiert. Die modellhafte Analyse der Daten zweier Kliniken hat gezeigt, dass fehlende oder extrem verkürzte Nachbeobachtungszeiten einen Einfluss auf die Schätzungen der Überlebenszeiten haben. Diese Verzerrungen sind durch statistische Korrekturen nicht behebbar, da die Verzerrungsquellen naturgemäß nur begrenzt bekannt sind. Um den großen Erfassungsaufwand zu reduzieren und Fehlerquellen zu minimieren, sollten Möglichkeiten geschaffen werden, dass die verschiedenen medizinischen Register ihre Datenbestände einfacher abgleichen und ergänzen können. Die beiden hier betrachteten Register werden in der Zukunft insbesondere ihre Bemühungen zur Sicherung der Nachbeobachtung stärker kombinieren.

Auch mit noch vorhandenen Erfassungsproblemen können Untersuchungen von Einflussfaktoren und zeitlichen Veränderungen innerhalb eines Registers weitgehend ihre Gültigkeit behalten. Vergleiche verschiedener Register oder Einrichtungen sollten aber mit besonderer Vorsicht und unter Hinzuziehung von Experten durchgeführt und interpretiert werden. Registerdaten auf eine komplexe Weise beeinflussen.

Limitierende Faktoren sind, dass sich die hier vorgestellten Ergebnisse nur auf die Analyse der Daten von zwei Kliniken der Stadt Magdeburg beziehen. Es wurde kein Populationsbezug angestrebt, sondern ein lokaler Datensatz mit für uns einsehbaren Erfassungsbedingungen betrachtet. Wir untersuchten die Auswirkung von fehlenden Patientendaten und unvollständiger und ggf. auch fehlerhafter Erfassung der Primär- und Verlaufsdaten auf die Voraussagen der Register. Auch muss darauf hingewiesen werden, dass die Überprüfung der Einträge des KKR durch das An möglicherweise dadurch verzerrt ist, dass es doch eine gewisse Abhängigkeit zwischen beiden Erfassungswegen gibt.
Konsequenz für Klinik und Praxis

- Patientendaten werden in Krebsregistern nie ganz vollständig erfasst. Die Erfassung hängt u. a. vom Krebs-Stadium ab.

- Schätzungen der Überlebenszeit aufgrund von Registerdaten können durch unvollständige Erfassung verzerrt werden.

- Um Fehlerquellen zu minimieren, müssen die Daten vollzählig, vollständig, richtig und aktuell sein. Dafür sollten die verschiedenen medizinischen Register versuchen, ihre Datenbestände abzugleichen und zu ergänzen. 


\section{Literatur}

1 Altendorf-Hofmann A. Überlebensraten als Instrument der Qualitätssicherung in Organzentren. Vortrag auf der 20. Informationstagung Tumordokumentation der klinischen und epidemiologischen Krebsregister, 10.-12. April 2013, Lübeck http://www.med.uni-giessen.de/akkk/info/20/ praesentationen/altendorf_hofmann.pdf Letzter Zugriff am 28.04.2015

2 Benedix F, Schmidt U, Mroczkowski P et al. Colon carcinoma - classification into right and left sided cancer or according to colonic subsite? - Analysis of 29, 568 patients. Eur J Surg Oncol. 2011; 37: 134-139

3 Eisemann N, Waldmann A, Katalinic A. Imputation of missing values of tumour stage in population-based cancer registration. BMC Med Res Methodol. 2011; 11 : 129

4 Gemeinsames Krebsregister der Länder Berlin, Brandenburg, Mecklenburg-Vorpommern, SachsenAnhalt und der Freistaaten Sachsen und Thüringen. Vollzähligkeit von Todesinformationen im GKR. In: GKR Kurz Informiert, Mai 2008, Ausgabe 2 www.berlin.de/gkr/_assets/lss_vollz.pdf Letzter Zugriff 11.05.2015

5 Gesetz zur Änderung des Gesundheitsdienstgesetzes vom 14.06.2000, GVBI LSA Nr. 21/2000

6 Hentschel S, Katalinic A (Hrsg.). Das Manual der epidemiologischen Krebsregistrierung. München: Zuckschwerdt-Verlag, 2008

7 Hofstädter F, Hölzel D. Was leisten Tumorregister für die Qualitätssicherung in der Onkologie? Der Onkologe 2008; 14: 1220-1233

8 Hölzel D, Eckel R, Engel J. Metastasierung beim kolorektalen Karzinom. Häufigkeit, Prognose und Folgerungen. Chirurg 2009; 80: 331-340

9 Klinkhammer-Schalke M, Marschner N, Hofstädter F. Register in der onkologischen Versorgungsforschung. Der Onkologe 2012; 18: 142-150

10 Robert Koch-Institut und die Gesellschaft der epidemiologischen Krebsregister in Deutschland e.V. (Hrsg). Krebs in Deutschland 2007/2008. 8. Ausgabe. Robert Koch-Institut (Hrsg) und die Gesellschaft der epidemiologischen Krebsregister in Deutschland e.V. (Hrsg). Berlin, 2012
11 Majek O, Gondos A, Jansen L et al. Sex Differences in Colorectal Cancer Survival: Population-Based Analysis of 164, 996 Colorectal Cancer Patients in Germany. PLoS ONE 2013; 8: e68077

12 Majek O, Gondos A, Jansen L et al. Survival from colorectal cancer in Germany in the early 21st century. Br J Cancer 2012; 106: 1875-1880

13 Mroczkowski P, Schmidt U, Sahm M et al. Prognostic factors assessed for 15, 096 patients with colon cancer in stages I and II. World J Surg 2012; 36: 1693-1698

14 Ptok H, Mundt A, Lippert H, Gastinger I. Rektumkarzinomchirurgie in Deutschland - eine 10-JahresÜbersicht auf der Grundlage der Daten des AN-Instituts für Qualitätssicherung in der operativen Medizin $\mathrm{gGmbH}$ an der Otto-von-Guericke-Universität Magdeburg. Zentralblatt Chirurgie 2013; 138: 418-426

15 Stabenow R, Wilsdorf-Köhler H, Neumeyer-Gromen A, Streller B. Krebsinzidenz und Krebsmortalität 2007-2008 im Erfassungsgebiet des GKR. In: Schriftenreihe des GKR, Berlin, 2012. Zusammenfassung

Interessenkonflikt

Prof. S. Kropf ist Arbeitsgruppenleiter Biometrie im gleichen Institut, in dem auch das klinische Krebsregister Magdeburg angesiedelt ist, Prof. J. Bernarding ist der Institutsdirektor.

Frau E. Burger ist Leiterin des klinischen Krebsregisters Magdeburg in diesem Institut.

Prof. K. Ridwelski und Prof. H. Lippert sind bzw. waren die Direktoren der beiden betrachteten chirurgischen Kliniken und sind Geschäftsführer bzw. wissenschaftlicher Leiter des An-Instituts für Qualitätssicherung in der operativen Medizin gGmbH an der Otto-von-Guericke-Universität Magdeburg.
DOI 10.1055/s-0041-102171

Dtsch Med Wochenschr 2015 140: e106-e113

(c) Georg Thieme Verlag KG . Stuttgart · New York . ISSN 0012-0472 